\title{
Obergurgl Research Conference Abstracts on "Cancer Stem Cells: Impact on Treatment"
}

Tyrol, Austria; 7-11 December 2016; Published: 16 November 2016

Correspondence to: Prof. Dr. Ira-Ida Skvortsova, Laboratory for Experimental and Translational Research on Radiation Oncology (EXTRO-Lab), Department of Therapeutic Radiology and Oncology, Innsbruck Medical University, Anichstr. 35, A-6020 Innsbruck, Austria.

E-mail: Ira.Skvortsova@i-med.ac.at; Ira.Skvortsova@tirol-kliniken.at

\section{A1}

\section{Proteomics identification of protein} biomarkers and signaling pathways for prostate cancer radioresistance therapy

Lei Chang ${ }^{1,2}$, Peter Graham ${ }^{1,2}$, Jingli Hao ${ }^{1,2}$, Valerie Wasinger $^{3,4}$, Jie $\mathrm{Ni}^{1,2}$, Julia Beretov ${ }^{1,2,5}$, Junli Deng ${ }^{1,2}$, Joseph Bucci ${ }^{1,2}$, David Malouf ${ }^{6}$, David Gillatt ${ }^{6,7}$, Yong Li $^{1,2}$

${ }^{1}$ Cancer Care Centre, St. George Hospital, Kogarah, Australia:

${ }^{2}$ St. George and Sutherland Clinical School, Faculty of Medicine, UNSW, Kensington, Australia;

${ }^{3}$ Bioanalytical Mass Spectrometry Facility, Mark Wainwright Analytical Centre, UNSW, Kensington, Australia;

${ }^{4}$ School of Medical Science, UNSW, Kensington, Australia;

${ }^{5}$ SEALS, Anatomical Pathology, St. George Hospital, Kogarah, Australia;

${ }^{6}$ Department of Urology, St. George Hospital, Kogarah, Australia;

${ }^{7}$ Australian School of Advanced Medicine, Macquarie University, Sydney, Australia

Background: Radioresistance is a major problem in prostate cancer $(\mathrm{CaP})$ radiotherapy $(\mathrm{RT})$. The mechanisms of $\mathrm{CaP}$ radioresistance are still unclear. We have recently developed CaP-RR (radioresistant) cell lines which display more aggressive characteristics including increased colony formation, invasion ability, sphere formation capability, and enhanced epithelial mesenchymal transition (EMT) and cancer stem cell (CSC) phenotypes. In addition, we found the PI3K/
Akt/mTOR pathway is closely linked with EMT and CSCs expression. Therefore, these CaP-RR cells, representative of the source of recurrence after RT, provide a very good model to mimic the clinical radioresistance condition to find biomarkers and signaling pathways for $\mathrm{CaP}$ radiotherapy.

Aim: The objective of this study was to identify candidate proteins and the main signaling pathways involved in $\mathrm{CaP}$ radioresistance, validate the identified potential biomarkers in CaP-radioresistant (RR) cell lines and animal xenografts, and perform the functional study from a selected candidate. Methods: The differential proteins from $\mathrm{CaP}$ parental cell lines (PC-3, DU145 and LNCaP) and CaP-RR sublines (PC-3RR, DU145RR and LNCaPRR) were analyzed using LC-MS/MS and identified by a label-free ion count approach. Pathways enriched as a result of radioresistance were assessed. Identified potential markers were validated in CaP-RR cell lines and subcutaneous (s.c) animal xenografts by Western blotting and immunohistochemistry. In addition, the protein fructose-bisphosphate aldolase A (ALDOA) was identified as a key protein in radioresistance and was selected for radiosensitivity study. Results: A total of 309 signaling pathway proteins were identified to be significantly different between CaP and CaP-RR cells 
( $P \leq 0.05$, fold differences $>1.5,>80 \%$ power). Among these proteins, nineteen are common among three paired $\mathrm{CaP}$ cell lines and associated with metastasis, progression, signaling pathways and radioresistance. The PI3K/Akt, VEGF, metabolism and ERK pathways were identified to be associated with $\mathrm{CaP}$ radioresistance. The expression of key proteins from the identified pathways was found to be significantly increased in CaP-RR cells and S.C animal xenografts compared to controls. Furthermore, the downregulation of ALDOA combined with RT effectively reduced colony capability, induced more apoptosis and increased radiosensitivity in CaP-RR cells. Conclusion: CaP radioresistance is associated with EMT and enhanced CSC phenotypes via activation of the PI3K/Akt/mTOR signaling pathway. CaP radioresistance is caused by multifactorial traits and several signaling pathways. Downregulation of ALDOA increases radiosensitivity in CaP-RR cells. Our findings indicate that interfering EMT/CSCs, ALDOA and signaling pathways, in combination with $\mathrm{RT}$ is promising for $\mathrm{CaP}$ radiotherapy.

\section{Key words:}

Prostate cancer, radiation therapy, radioresistance, cancer stem cell, LC-MS/MS, signaling pathway

\section{A2}

\section{Tumor-associated fibroblast-conditioned medium induces CDDP resistance in HNSCC cells}

Teresa Bernadette Steinbichler, Jozsef Dudas, Herbert Riechelmann

Medical University of Innsbruck, Innsbruck, Austria

Aim: EMT contributes to tumor progression and metastasis. We aimed to investigate the effects of EMT on Cisplatin resistance in HNSCC (head and neck squamous cell carcinoma)-cells. Methods: EMT was induced in HNSCC cells using conditioned medium from a tumor cell/fibroblast co culture and confirmed with vimentin and $E$ cadherin expression analysis at RNA and protein level. The tumor cells were alternatively treated with $1 \mathrm{ng} / \mathrm{mL}$ TGF- $\beta 1$. The response to Cisplatin was evaluated with viability and clonogenic assays. Results: Treatment with conditioned medium induced a mesenchymal phenotype and increased the viability of the tumor cells. Moreover, it doubled the IC50 of Cisplatin of SCC-25 cells from $6.2 \mu \mathrm{mol} / \mathrm{L}$ to $13.1 \mu \mathrm{mol} / \mathrm{L}(P<0.001)$. The IC50 of Cisplatin of Detroit 562 cells was increased following treatment with conditioned medium from $13.1 \mu \mathrm{mol} / \mathrm{L}$ to 26.8 $\mu \mathrm{mol} / \mathrm{L}(P<0.01)$. Treatment with TGF- $\beta 1$ induced similar phenotypic changes as co-culture conditioned medium, but decreased tumor cell viability and did not alter Cisplatin resistance. Conclusion: Cell free medium from an epithelial tumor cell/fibroblast coculture was able to induce EMT in HNSCC cells. Co-culture treated HNSCC cells revealed increased viability and were less sensitive to Cisplatin treatment. TGF- $\beta 1$ also induced a mesenchymal phenotype, but decreased tumor cell viability and did not alter resistance to CDDP in HNSCC cells.

\section{Key words:}

Cisplatin resistance, tumor-associated fibroblasts, tumor microenvironment, EMT

\section{A3}

\section{Identifying metabolic biomarkers of paediatric glioma cancer stem cells in tumour development and drug resistance}

\author{
Alice Agliano, Maria Vinci, Chris Jones, Gabriela Kramer- \\ Marek, Martin Leach, Nada Al-Saffar
}

The Institute of Cancer Research, London, UK

Background: Paediatric glioblastoma multiforme (pGBM) is one of the most aggressive forms of cancer of the central nervous system in children. There is increasing evidence that cancer stem cells (CSC) can contribute to the current poor outcome of pGBM since CSC play an important role in tumour initiation and drug resistance. Much effort has been directed at identifying biomarkers able to recognize and select CSC. However, this has proven challenging due to their continuous evolution during tumour progression. Metabolism has been recognized as an important regulator of several functions in stem cells and even though metabolic aspects of tumour development are widely studied, little is known about CSC metabolism. Nuclear magnetic resonance (NMR) and positron emission tomography (PET) are powerful non-invasive imaging tools that can be used to evaluate aspects of tumour cell and CSC metabolism.

Aim: To characterise metabolic differences between CSCs and non-CSCs that are detectable by NMR and PET and to determine how cell signalling pathways alter CSC metabolism in order to identify possible therapeutic targets to develop CSC-targeted therapies for pGBMs. Methods: Cancer cell lines with stem-like features (CSLC) have been created following culture of a panel of paediatric cell lines, such as SF188 and KNS42, and primary cells on a laminin substrate with specific CSC media supplemented with growth factors. CSLC cell lines have been compared to the correspondent parental cell line (non-CSLC) grown 
under standard culture conditions. Metabolism was evaluated by NMR and radionuclide uptake. Results: In vitro $1 \mathrm{H}$ NMR and 18F-FDG-PET uptake studies showed that newly established pGBM CSLC express a different metabolic signature compared with non-CSLC cell lines. Differences have been observed in the levels of several metabolites, including lactate, glutamine and several lipids involved in the membrane turnover such as phosphocholine, glycerophosphocholine and cholesterol. These findings correlated with changes in the expression of metabolism- and cell divisionassociated proteins and genes. The increased gene expression of the glycolytic enzymes LDH-A, HK2, and Glut-1, suggested that the CSLCs rely mainly on aerobic glycolysis. This increased reliance on glucose metabolism together with a low mitochondrial activity reduced the levels of ROS in CSLCs, a feature that has been associated with EMT and pluripotency. Moreover, contrary to non-CSLCs, treatment with the PI3K/mTOR inhibitor NVP-BEZ235 did not affect cell viability or changes CSLCs metabolic signature, indicating induced drug resistance of the CSLCs upon this treatment. Conclusion: We have shown that pGBM CSLCs have different metabolic features from non-CSLCs. Improved understanding of mechanisms related to CSC drug resistance to $\mathrm{PI} 3 \mathrm{~K} / \mathrm{mTOR}$ inhibitors could guide the identification of potential targets, leading to development of more effective treatments for pGBM.

\section{Key words:}

Cancer stem cells, metabolic biomarker, paediatric glioblastoma multiforme, nuclear magnetic resonance, positron emission tomography, drug resistance

\section{A4 \\ Cancer stem cells in melanoma: a complex problem}

\section{Caterina A.M. La Porta}

University of Milan, Milan, Italy

Cancer progression in humans is difficult to infer because we do not routinely sample patients at multiple stages of their disease. The identification of cancer stem cell (CSC) subpopulations inside tumors opens a new perspective on cancer development, since it implies that tumors can only be eradicated by targeting CSCs. Several markers have been proposed in the literature to identify CSCs both in breast and melanoma but no consensus has been reached, leading to the hypothesis that the CSC phenotype might be dynamically switched. Herein we provide quantitative evidence of CSCs in melanoma discussing the complex network regulating their biological functions.

\section{Key words:}

Cancer stem cells, melanoma, complexity, miRNA

\section{A5 \\ Low extracelluar pH inhibits glycolysis and decreases transcription factor activity responsible for stemness in induced pluripotent stem cells}

Anja Wilmes ${ }^{1}$, Caroline Rauch ${ }^{1}$, Giada Carta ${ }^{1}$, Georg Kern'1, Florian Meier ${ }^{2}$, Wilfried Posch ${ }^{1}$, Doris Wilflingseder ${ }^{1}$, Lyle Armstrong $^{3}$, Majlinka Lako ${ }^{3}$, Mario Beilmann², Gerhard Gstraunthaler ${ }^{1}$, Paul Jennings ${ }^{1}$

${ }^{1}$ Medical University of Innsbruck, Innsbruck, Austria;

${ }^{2}$ Boehringer Ingelheim, Ingelheim, Germany;

${ }^{3}$ University of Newcastle, Newcastle, UK

Induced pluripotent stem cells (iPSC) have the potential to revolutionize biological experimentation and thus the uptake of this new technology is widespread. However, culturing iPSC is both time consuming and expensive as they require daily medium exchange. Our study investigates the reason for this high demand on frequent medium replacement.

Two human iPSC lineages were fed at different intervals up to $72 \mathrm{~h}$ either in a full growth area (FGA) or a restricted growth area (RGA). The FGA consisted of a well of a 6 well plate coated with Matrigel ${ }^{\mathrm{TM}}$ and the RGA consisted of a coated coverslip placed in a well. Medium was sampled every $24 \mathrm{~h}$ and glucose, lactate, $\mathrm{pH}$ were measured. In addition, flow cytometry was employed to investigate cell cycle alterations and TransAM assays utilized for CMYC, FOXO1 and p53 activity.

FGA cultured iPSC that were not fed every $24 \mathrm{~h}$ had significantly reduced growth rates by day 2 and showed increasing cell death by day 3 . In contrast, RGA cultured cells grew to confluence over 3 days. Surprisingly, glucose was not exhausted under any condition. Instead, the extracellular $\mathrm{pH}$ reached 6.8 after $72 \mathrm{~h}$ in FGA cultures. Reducing medium $\mathrm{pH}$ to 6.8 also inhibited glycolysis, initiated a cell cycle block in G0/G1 and decreased in CMYC and FOXO-1 transcriptional activity.

This study demonstrates that iPSC are susceptible to cell culture medium acidification, a likely limiting factor in maintenance of proliferative and pluripotent status. Culturing iPSC in RGA prevents rapid extracellular acidification, by limiting cell numbers, while still maintain optimal oxygen diffusion rates and allows 
longer feeding cycles whilst still ensuring pluripotency. These results may provide critical information for scale up procedures, e.g. the use of bioreactors, careful control of extracellular $\mathrm{pH}$ will be important.

Key words:

iPSC, pH, glucose, FOXO1, cMYC, growth arrest

\section{A6}

\section{Mechanisms of radioresistance in prostate cells}

Fabian Guggenberger ${ }^{1}$, Holger Erb², Ira-Ida Skvortsova ${ }^{3}$, Zoran Culig ${ }^{1}$, Frédéric R. Santer ${ }^{1}$

${ }^{I}$ Division of Experimental Urology, Medical University of Innsbruck, Innsbruck, Austria;

${ }^{2} Y C R$ Cancer Research Unit, Department of Biology, University of York, York, UK;

${ }^{3}$ Department of Therapeutic Radiology and Oncology, Medical University of Innsbruck, Innsbruck, Austria

Background: Prostate cancer ( $\mathrm{PCa}$ ) is one of the most commonly diagnosed malignancies in men in Western nations. Among androgen deprivation therapy (ADT), radiation therapy is an approved treatment either for early stage local PCa, but also for metastatic M1 stage PCa. However, tumour relapse is a frequent event that affects about $80 \%$ of patients undergoing prior treatment. There is increasing evidence that the occurrence of cancer stem cells (CSC) may play an important role in therapy resistance, in particular also in radioresistance. The occurrence of CD133-positive CSCs within the basal, less differentiated layer of the malignant prostatic epithelium was demonstrated. Those cells were shown to have increased colony forming efficacy and less double-strand breaks after irradiation due to a higher DNA repair capacity when compared to more differentiated populations. Moreover, aldehyde dehydrogenase 1 expressing (ALDH1+) cells derived from $\mathrm{PCa}$ cell lines were shown to be more radioresistant than ALDH1- cells and ALDH1 inhibition lead to increased radiosensibility. However, our knowledge on the mechanisms of radioresistance of the prostatic basal layer is still limited.

Aim: The aim of this study is to identify a novel molecular mechanism underlying radioresistance in the prostate basal cell layer containing stem cells. Approach: To investigate the effect of irradiation exposure on prostate basal cells we irradiated benign PrEPs and an immortalised benign, basal prostatic cell line (EP156T) for 21 times following a therapeutic schedule with either $0,0.5$ or 1 Gray for 5 times per week using a linear particle accelerator (LINAC). Total RNA was isolated and NextGen transcriptome sequencing followed by bioinformatical analysis (including pathway analysis) and literature research will be performed. Finally, a regulated candidate gene will be chosen for further experiments. Methods/ Results: PrEPs obtained from 5 patients receiving radical prostatectomy were successfully isolated via collagenase digestion from prostate tissue specimens and cultured in the presence of a feeder layer. PrEPs and EP156T have been characterised by clonogenic assays and by label retention assay using FACS analysis subsequent to PKH67 membrane labelling, which might indicate the presence of stem cells within the cultures. The repeated exposition to irradiation using the protocol described above for both, PrEPs and EP156T cells, is finished and gained total RNA from the cultures will be sequenced by the company Microsynth. Bioinformatical analysis will be done in collaboration with the cancer Computational Biology Center (Erasmus MC, Rotterdam, NL). Outlook: Once the bioinformatical analysis is finished a careful review of literature is performed. Based thereon a candidate gene, whose expression is shown to be strongly altered by irradiation, is chosen for further experiments (e.g. clonogenic assay, knock down, over-expression, inhibition) that will test the candidate's involvement in radioprotection and which may have the potential as a possible target for radiosensibilisation. This may improve co-treatment strategies for future irradiation therapy.

\section{Key words:}

Prostate cancer, cancer stem cells, primary cells, radioresistance

\section{A7}

\section{Cysteine cathepsins and their inhibitors as regulators of cancer stem cell dormancy and differentiation}

Janko Kos ${ }^{1,2}$, Milica Perišič Nanut ${ }^{2}$, Mateja Prunk ${ }^{2}$, Urša Pečar Fonović ${ }^{1}$, Anja Pišlar ${ }^{1}$, Ana Mitrović ${ }^{1}$, Jerica Sabotič², Špela Magister ${ }^{2}$, Anahid Jewett ${ }^{3}$

${ }^{I}$ University of Ljubljana, Faculty of Pharmacy, Ljubljana, Slovenia; ${ }^{2}$ Jožef Stefan Institute, Department of Biotechnology, Ljubljana, Slovenia; ${ }^{3}$ University of California, School of Dentistry, Los Angeles, USA

Cysteine cathepsins are lysosomal peptidases involved in different processes of tumor development and progression. There is increasing evidence that these enzymes may regulate also homeostasis and differentiation of cancer stem cells. In particular, cysteine cathepsins $K$ and $X$ were shown to be involved in cytokine-induced niche dormancy as well as in mobilization process that release cancer stem cells from their niches. Cathepsin $X$ has been proposed to participate in proteolytic processing of CXCL-12 
chemokine (SDF-1), which is involved in adhesion and migration of cancer stem cells. Additionally, cathepsin X cleaves C-terminal ends of enolase and profilin, another two proteins that were suggested to alter cancer stem cells adhesion, migration and their metastatic potential. Cathepsin $\mathrm{X}$ also cleaves beta-2 chain in Mac-1 and LFA-1 integrin receptors and binds to alpha-v-beta 5 integrin receptor via RGD motif in the pro-region, affecting in this way the adhesion of endothelial and tumor cells and presumably cancer stem cells. Cathepsin $X$ knock out accelerates a progress to senescence in vitro and in vivo via $\mathrm{p} 16$, p21 and p53 signaling pathway.

The activity of cysteine cathepsins is regulated by endogenous protein inhibitors cystatins. Of these, cystatin $F$ is the only cystatin that is localized in endosomal/lysosomal vesicles. In cytotoxic T cells and NK cells its main role is the control of progranzyme convertase activity of cathepsins $\mathrm{C}$ and $\mathrm{H}$ and consequently, the granzyme dependent cytotoxic function. It is known that cytotoxic function of NK cells is suppressed after their interaction with tumor cells or cancer stem cells, the status is termed split anergy. The mechanism includes cytokine cross-talk, however, target cells may also secrete inactive dimeric cystatin $F$ which after internalization to NK cells enters endosomal/lysosomal vesicles and after activation/ monomerisation inhibits cathepsins $\mathrm{C}$ and $\mathrm{H}$ and down-regulates cell cytotoxicity. Anergized NK cells cause differentiation of cancer stem cells by secreted cytokines and as a result, differentiated tumors become resistant to NK cell-mediated cytotoxicity.

Our results show that some of cysteine cathepsins and their endogenous inhibitors have specific roles in cancer stem cell functions designating them as potential therapeutic targets for improving anticancer therapy.

\section{Key words:}

Cathepsin, cystatin, anergy, SDF-1, profilin, enolase

\section{A8}

\section{Hypoxia and expression levels of cancer stem cell markers are prognostic for loco- regional control after radiochemotherapy in locally advanced head and neck squamous cell carcinoma}

Fabian Lohaus ${ }^{1,9}$, Annett Linge ${ }^{1,10}$, Steffen Löck ${ }^{10}$, Volker Gudziol $^{19}$, Alexander Nowak ${ }^{20}$, Cläre von Neubeck ${ }^{1,10}$, Inge Tinhofer ${ }^{2,11}$, Volker Budach ${ }^{2,11}$, Ali Sak ${ }^{3,12}$, Martin Stuschke ${ }^{3,12}$, Panagiotis Balermpas ${ }^{4,13}$, Claus Rödel ${ }^{4,13}$, Melanie Avlar ${ }^{5,14}$, Anca-Ligia Grosu ${ }^{5,14}$, Amir Abdollahi6, ${ }^{6,15}$,
Jürgen Debus ${ }^{6,15}$, Claus Belka ${ }^{7,16}$, Steffi Pigorsch ${ }^{7,17}$, Stephanie E. Combs ${ }^{7,17}$, David Mönnich ${ }^{8,18}$, Daniel Zips ${ }^{8,18}$, Gustavo B. Baretton ${ }^{1,21}$, Frank Buchholz ${ }^{1,22}$, Michael Baumann ${ }^{1,9}$, Mechthild Krause ${ }^{1,9}$

${ }^{I}$ German Cancer Research Center (DKFZ), Heidelberg and German Cancer Consortium (DKTK) partner site: Dresden, Germany;

${ }^{2}$ German Cancer Research Center (DKFZ), Heidelberg and German Cancer Consortium (DKTK) partner site: Berlin, Germany;

${ }^{3}$ German Cancer Research Center (DKFZ), Heidelberg and German Cancer Consortium (DKTK) partner site: Essen, Germany;

${ }^{4}$ German Cancer Research Center (DKFZ), Heidelberg and German Cancer Consortium (DKTK) partner site: Frankfurt, Germany;

${ }^{5}$ German Cancer Research Center (DKFZ), Heidelberg and German Cancer Consortium (DKTK) partner site: Freiburg, Germany;

${ }^{6}$ German Cancer Research Center (DKFZ), Heidelberg and German Cancer Consortium (DKTK) partner site: Heidelberg, Germany;

${ }^{7}$ German Cancer Research Center (DKFZ), Heidelberg and German Cancer Consortium (DKTK) partner site: Munich, Germany;

${ }^{8}$ German Cancer Research Center (DKFZ), Heidelberg and German Cancer Consortium (DKTK) partner site: Tübingen, Germany;

${ }^{9}$ Universitätsklinikum Carl Gustav Carus der TU Dresden, Dresden, Germany;

${ }^{10}$ OncoRay - National Center for Radiation Research in Oncology, Faculty of Medicine and University Hospital Carl Gustav Carus, Technische Universität Dresden, Dresden, Germany;

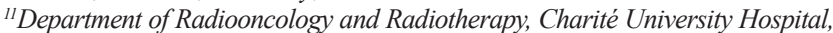
Berlin, Germany;

${ }^{12}$ Department of Radiotherapy, Medical Faculty, University of Duisburg-Essen, Essen, Germany;

${ }^{13}$ Department of Radiotherapy and Oncology, Goethe-University Frankfurt, Frankfurt, Germany;

${ }^{14}$ Department of Radiation Oncology, University of Freiburg, Freiburg, Germany; ${ }^{15}$ Heidelberg Institute of Radiation Oncology (HIRO), National Center for Radiation Research in Oncology (NCRO), University of Heidelberg Medical School and German Cancer Research Center (DKFZ), Heidelberg, Germany;

${ }^{16}$ Department of Radiotherapy and Radiation Oncology, Ludwig-MaximiliansUniversität, Munich, Germany;

${ }^{17}$ Department of Radiation Oncology, Technische Universität München, Munich, Germany;

${ }^{18}$ Department of Radiation Oncology, Faculty of Medicine and University Hospital Tübingen, Eberhard Karls Universität Tübingen, Tübingen, Germany; ${ }^{19}$ Department of Otorhinolaryngology, Faculty of Medicine and University Hospital Carl Gustav Carus, Technische Universität Dresden, Dresden, Germany;

${ }^{20}$ Department of Oral and Maxillofacial Surgery, Faculty of Medicine and University Hospital Carl Gustav Carus, Technische Universität Dresden, Dresden, Germany;

${ }^{21}$ Institute of Pathology, Faculty of Medicine and University Hospital Carl Gustav Carus, Technische Universität Dresden, Dresden, Germany;

${ }^{22}$ University Cancer Center (UCC), Medical Systems Biology, University Hospital Carl Gustav Carus, Technische Universität Dresden, Dresden, Germany

Aim: The outcome after curative treatment of locally advanced head and neck squamous cell carcinoma (LA-HNSCC) remains unsatisfactory. Except of HPV-infection status there is no further established biomarker for treatment stratification. Currently, treatment decisions are mainly based on the tumor site and the TNM system regardless of the heterogeneous biology of HNSCC. For identification and validation of biomarkers, the German Cancer Consortium Radiation Oncology Group (DKTK-ROG) initiated a multicenter retrospective/prospective biomarker trial to explore their impact on locoregional control (LRC) after postoperative (PORT-C) and primary radiochemotherapy. Methods: In the multicenter retrospective part of the study, 355 patients with HNSCC of the oral cavity, oro- and hypopharynx were included. All patients received 
cisplatin-based radiochemotherapy (RCTx) between 2005 and 2012. The postoperative cohort consisted of 195 patients, who were all treated with PORT-C because of clinical high risk parameter. The second cohort consisted of 160 patients treated with primary RCTx. FFPE-material, radiotherapy treatment plans and images were centrally collected. Tumor volume was segmented on CT-based radiotherapy treatment plans. HPV status (p16 overexpression) and CD44 expression were analysed by immunohistochemistry. Gene expression analyses were performed for hypoxiaassociated genes and the potential cancer stem cell (CSC) markers SLC3A2, MET and CD44. Results of the biomarker analyses, clinical parameters and tumor volume were correlated with the clinical outcome. Primary endpoint was LRC. Results: Multivariate analysis (MVA) revealed the impact of hypoxia and expression of CSC markers in HPV(-) HNSCC on LRC after PORT-C (hypoxia gene signature: HR 4.54, $P=$ 0.006; MET: HR 3.71, $P=0.016$; SLC3A2: HR 8.54, $P$ $=0.037$; CD44: HR 3.36, $P=0.054$ ). For primary RCTx a significant impact of tumor volume, HPV status and expression of CSC markers (tumor volume: HR 2.63, $P$ $=0.003$, SLC3A2: HR 2.03, $P=0.021 ; \mathrm{HPV}: \mathrm{HR} 0.35, P$ $=0.086)$ on LRC was seen in MVA. A significant impact of hypoxia associated gene expression was only seen in small tumors $(<25 \mathrm{ccm})$ (HR 9.2, $P=0.38)$. Conclusion: We demonstrated that the expression of CSC markers and hypoxia-associated genes are prognosticators for LRC in addition to the HPV-infection status in patients suffering from LA-HNSCC, who were treated with PORT-C or primary RCTx. After validation of these promising results in the currently ongoing part of the prospective trial of the DKTK-ROG, along with established clinical parameters, they may help to further stratify patients for individualized escalation and de-escalation strategies.

\section{Key words:}

HNSCC, PORT-C, hypoxia, radiochemotherapy, biomarker

\section{A9}

\section{Epidermal growth factor receptor activity is elevated in glioma cancer stem cells and is required to maintain chemotherapy and radiation resistance}

Lisa Y. Pang, Lauren Saunders, David J. Argyle

University of Edinburgh, Edinburgh, UK

Glioblastoma remains among the most aggressive of all human and canine malignancies, displaying high mortality rates and limited treatment options.
We propose that given the similarities between canine and human gliomas, such as incidence of occurrence, histopathology, molecular characteristics, and response to therapy, that canine gliomas are a natural model of the human disease. A range of human and canine tumours have been shown to harbor specific subpopulations of cells with stem celllike properties that initiate and maintain neoplasticity while resisting conventional therapies. Here, we show that both canine and human glioma cell lines contain a small population of cancer stem cells (CSCs), and by molecular profiling highlight the important role of the epidermal growth factor receptor (EGFR) pathway in canine CSCs. EGFR signaling is crucial in the regulation of cancer cell proliferation, migration and survival. To date EGFR-targeted interventions alone have been largely ineffective. Our findings confirm that specifically inhibiting EGFR signaling alone has no significant effect on the viability of CSCs. However inhibition of EGFR did enhance the chemo- and radiosensitivity of both canine and human glioma CSCs, enabling this resistant, tumourigenic population of cells to be effectively targeted by conventional therapies.

\section{Key words:}

Glioma, cancer stem cells, comparative oncology, EGFR

\section{A10}

\section{MET inhibition overcomes radiation resistance of glioblastoma stem-like cells}

Francesca De Bacco1, Antonio D'Ambrosio', Elena Casanova1, Francesca Orzan ${ }^{1}$, Roberta Neggia ${ }^{1}$, Raffaella Albano1, Federica Verginelli', Manuela Cominelli², Pietro L. Poliani ${ }^{2}$, Paolo Luraghi', Gigliola Reato', Serena Pellegatta ${ }^{3}$, Gaetano Finocchiaro ${ }^{3}$, Timothy Perera $^{4}$, Elisabetta Garibaldi ${ }^{1}$, Pietro Gabriele ${ }^{1}$, Paolo M. Comoglio', Carla Boccaccio ${ }^{1}$

${ }^{I}$ Candiolo Cancer Institute, FPO-IRCCS, University of Torino, Torino, Italy; ${ }^{2}$ Department of Molecular and Translational Medicine, University of Brescia, Brescia, Italy;

${ }^{3}$ Unit of Molecular Neuro-Oncology, Fondazione IRCCS Istituto Neurologico C. Besta, Milano, Italy;

${ }^{4}$ Octimet Oncology Ltd., Oxford, UK

Glioblastoma (GBM), the most aggressive and common primary brain tumor, usually remains refractory to the best standard of care, entailing radiotherapy as a mainstay, and, often, as the only treatment option. GBM radioresistance has been associated with distinctive properties of the GBM stem-like subpopulation (GSC): after irradiation, while bulk-cells accumulate DNA damage and die, stem-like cells efficiently activate DNA repair mechanisms and survive, driving tumor recurrence. A deeper understanding of the mechanisms 
of GSC radioresistance is needed, in order to identify druggable targets for radiosensitization and long-term effective therapeutic response.

By analyzing a large panel of GSCs propagated in vitro as neurospheres, we provide evidence that radioresistance is significantly higher in GSCs than in their differentiated counterpart (including cells derived from GSC pseudodifferentiation). We show that the levels of radioresistance are similar in GSCs displaying different genetic alterations or transcriptional profiles, which are characteristic of distinct GBM subtypes (classical, proneural, mesenchymal). However, in a subset of neurospheres, radioresistance is associated with expression of MET, the HGF tyrosine kinase receptor. MET expressing GSCs are positively selected by ionizing radiation in vitro and, possibly, also in vivo, as assessed in a cohort of human patients including 20 cases of surgically removed primary GBMs and their matched recurrences.

We elucidate that MET promotes GSC radioresistance through a novel mechanism, relying on AKT activity and leading to (i) sustained activation of Aurora kinase A, ATM kinase, and the downstream effectors of DNA repair; (ii) phosphorylation and cytoplasmic retention of p21, which is associated with anti-apoptotic functions. We show that MET pharmacological inhibition causes DNA damage accumulation in irradiated GSCs, and their depletion in vitro and in GBMs generated by GSC xenotransplantation. Preclinical evidence is thus provided that MET inhibitors can radiosensitize tumors and convert GSC positive selection, induced by radiotherapy, into GSC eradication.

\section{Key words:}

Glioblastoma, glioblastoma stem-like cells, MET oncogene, MET inhibitor, radiotherapy, radiosensitization

\section{A11}

\section{Transmembrane protein as potential CD9 is glioblastoma stem cell theranostic}

\author{
Tamara Lah Turnšek \\ National Institute of Biology, Faculty of Chemistry and Chemical \\ Engineering, University of Ljubljana, Ljubljana, Slovenia
}

Glioblastomas, the most agreesive brain tumour, is presumably maintained by a sub-population of stemlike tumor cells (GSC) that divide asymmetrically, sustaining pool of highly stable stem cells, resisting therapy. Targeting these cells thus represents more selective approach a need to define specific markers that characterize GSC. In the present study, we performed transcriptomic analysis of glioblastoma tissues compared to normal brain tissues revealing sensible up-regulation of CD9 gene. ${ }^{[1]}$ CD9 encodes the transmembrane protein tetraspanin which is involved in tumor cell invasion, apoptosis and resistance to chemotherapy. We validated CD9 gene and protein expression showing selective up-regulation in GSC from primary biopsies and in primary organotypic glioblastoma spheroids as well as in U87-MG and U373 glioblastoma cell lines, whereas no or low CD9 gene expression was observed in their normal counterparts. CD9 silencing in three CD133+ subtypes of GSC lines ${ }^{[2]}$ (NCH644, NCH421k and NCH660h) led to decreased cell proliferation, survival, invasion, and self-renewal ability, and altered expression of the stem-cell markers CD133, nestin and SOX2. Moreover, CD9-silenced glioblastoma stem cells showed altered kinase signaling patterns. Orthotopic xenotransplantation of CD9-silenced GSC into nude rats promoted prolonged survival. Finally using the public REMBRANDT database for brain tumors, we confirmed the prognostic value of CD9, whereby a more than two fold up-regulation correlates with shorter patient survival. Therefore, we propose CD9 for further evaluation as a target for GBM treatment.

\section{Key words:}

Biomarker, CD9, glioblastoma stem cells, neural stem cells, tetraspanin

\section{References}

1. Podergajs N, Motaln H, Rajčević U, Verbovšek U, Koršič M, Obad N, Espedal H, Vittori M, Herold-Mende C, Miletic H, Bjerkvig R, Turnšek TL. Transmembrane protein CD9 is glioblastoma biomarker, relevant for maintenance of glioblastoma stem cells. Oncotarget 2016;7:593-609.

2. Podergajs N, Brekka N, Radlwimmer B, Herold-Mende C, Talasila KM, Tiemann K, Rajcevic U, Lah TT, Bjerkvig R, Miletic H. Expansive growth of two glioblastoma stem-like cell lines is mediated by bFGF and not by EGF. Radiol Oncol 2013;47:330-7.

\section{A12}

\section{Cancer stem cells: the seeds for recurrent ovarian cancer}

\author{
Nuzhat Ahmed ${ }^{1,2}$, Emily Chan ${ }^{3}$, Chantel Samardjiza ${ }^{3}$, \\ Khalid Abubaker ${ }^{4}$, Ardian Latifi ${ }^{4}$, George Kannourakis ${ }^{1,2}$, \\ Jock Findlay ${ }^{5}$ \\ ${ }^{I}$ Fiona Elsey Cancer Research Institute, Victoria, Australia; \\ ${ }^{2}$ Federation University Australia, Ballarat, Australia; \\ ${ }^{3}$ Department of Obstetrics and Gynaecology, University of Melbourne, \\ Victoria, Australia; \\ ${ }^{4}$ Department of Surgery, University of Melbourne, Victoria, Australia; \\ ${ }^{5}$ Hudson Institute of Medical Research, Clayton, Victoria, Australia
}

Aim: The treatment of ovarian cancer (OC) with chemotherapy leaves resistant cancer cells which in 
a short time re-grow as recurrent cancer. A diverse array of resistance mechanisms for chemotherapy has been described but none have proven to be viable targets in a clinical setting. Cancer stem cells (CSCs) are increasingly accepted as the putative mediators of chemoresistance and relapse of cancer. This study aimed to understand the molecular mechanisms involved with chemoresistance and recurrence by investigating the roles of CSCs and their associated pathways in OC cell lines and tumor cells isolated from the ascites of $\mathrm{OC}$ patients obtained prior (chemonaive, $\mathrm{CN}$ ) to and after chemotherapy treatment (recurrent, $\mathrm{CR}$ ). Methods: Ascites collected from $\mathrm{CN}$ and $\mathrm{CR}$ OC patients diagnosed with advanced-stage serous OC were cultured using a novel in vitro method to obtain a distinct population of epithelial tumor cells. Flow cytometry and immunofluorescence were used to characterize the tumor population. High-resolution label-free quantitative proteomic profiling was used to define significantly differentially expressed proteins between $\mathrm{CN}$ and $\mathrm{CR}$ tumor cells. KEGG and DAVID software's were used to determine pathways associated with CR cells. The mechanisms of survival of in vitro cisplatin or paclitaxel treated ascites-derived tumor cells as well as cultured OC cell lines were determined by in vitro assays and in mouse xenografts. In another approach, the expression of embryonic stem cell factor Oct4A in primary OC tumors as well as $\mathrm{CN}$ and $C R$ ascites-derived tumor cells was determined by qPCR. The functional role of Oct4A was investigated using in vitro assays and in vivo mouse models with stable knockdown (shRNA) of Oct4A in an OC cell line. Results: Proteomic profiling of $\mathrm{CN}$ and $\mathrm{CR}$ tumor cells showed significant differences in proteins encoding for immune surveillance, DNA repair mechanisms, cytoskeleton rearrangement, cell-cell adhesion, cell cycle pathways, cellular transport, and proteins involved with glycine/proline/arginine synthesis in tumor cells isolated from CR relative to $\mathrm{CN}$ patients. Pathway analyses revealed enrichment of metabolic pathways, DNA repair mechanisms and energy metabolism pathways in CR tumor cells. The treatment of ascites-derived OC cells with chemotherapy in vitro resulted in a CSC-like residual population with increased activation of JAK2/STAT3 pathway. Both JAK2/STAT3 activation and CSC-like characteristics were suppressed by a low dose JAK2 specific inhibitor, Momelotinib, in vitro and in vivo. This also resulted in a significantly reduced tumor burden, increased disease-free survival periods in mice in vivo. In another approach, stable knockdown of Oct4A resulted in the decreased expression of CSCs in OC cells and was consistent with decreased cell proliferation, migration and chemoresistance in vitro. In vivo Oct4A knockdown cells produced a significantly reduced tumor burden in mice resulting in a significantly increased survival period compared to vector control cells. Conclusion: The above studies suggest that targeting the CSCs may prove a therapeutic option for advanced-stage OC patients.

\section{Key words:}

Ovarian cancer, metastasis, ascites, cancer stem cells, chemoresistance, recurrence

\section{A13 \\ Targeting of the epigenetic reprogramming for prostate cancer cell radiosensitization}

\author{
Anna Dubrovska ${ }^{1,2,3}$, Monica Cojoc ${ }^{1}$, Linda Hein ${ }^{1}$, Anna \\ Tyutyunnykova ${ }^{1}$, Claudia Peitzsch ${ }^{1}$
}

${ }^{I}$ OncoRay-National Center for Radiation Research in Oncology, Medical Faculty and University Hospital Carl Gustav Carus, Technische Universität Dresden and Helmholtz-Zentrum Dresden-Rossendorf, Dresden, Germany; ${ }^{2}$ German Cancer Consortium (DKTK) Dresden, Dresden, Germany;

${ }^{3}$ German Cancer Research Center (DKFZ) Heidelberg, Heidelberg, Germany

Radiation therapy is one of the mainstays of curative prostate cancer treatment. Nevertheless, the doses needed to eradicate prostate cancer are very high bearing the potential of side effects in normal tissues, and the risk of recurrence after radiotherapy still remains substantial in locally advanced disease. Tumor relapse after radiotherapy is attributed to the population of cancer stem cells (CSCs) which survived the treatment. Therefore, analysis of the CSC populations might be an important predictive tool for radiotherapy and individualized treatment selection. However, compelling evidence suggests a high plasticity of CSCs imposed by tumor treatment. Our study revealed that irradiation causes long-term upregulation in the expression of stem cell markers and induces tumor cell reprogramming. Furthermore, radioresistant and tumorigenic cell populations undergo a phenotypic switch during the course of radiotherapy. This phenotypic plasticity is associated with genetic and epigenetic changes induced by irradiation. Our results indicate that irradiation drives methylation of histone $\mathrm{H} 3$ on the promoter sequence of aldehyde dehydrogenase 1A1 (ALDH1A1) leading to the activation of gene transcription. We found that inhibition of $\mathrm{H} 3$ methylation with DZNep triggers apoptosis and inhibits tumorigenicity of the radioresistant prostate cancer cells as well as leads to their radiosensitization. Our studies suggest that radioresistant properties of prostate cancer cells are dynamic in nature and that combination of irradiation with therapeutic agents which prevent tumor cell reprogramming may enhance the effectiveness of treatment. 
Key words:

Radiation therapy, cancer stem cells, epigenetic reprogramming, radiosensitization

\section{References}

1. Peitzsch C, Cojoc M, Hein L, Kurth I, Mäbert K, Trautmann F, Klink B, Schröck E, Wirth MP, Krause M, Stakhovsky EA, Telegeev GD, Novotny V, Toma M, Muders M, Baretton GB, Frame FM, Maitland NJ, Baumann M, Dubrovska A. An epigenetic reprogramming strategy to resensitize radioresistant prostate cancer cells. Cancer Res 2016;76:2637-51.

2. Cojoc M, Peitzsch C, Kurth I, Trautmann F, Kunz-Schughart LA, Telegeev GD, Stakhovsky EA, Walker JR, Simin K, Lyle S, Fuessel S, Erdmann K, Wirth MP, Krause M, Baumann M, Dubrovska A. Aldehyde dehydrogenase is regulated by $\beta$-Catenin/TCF and promotes radioresistance in prostate cancer progenitor cells. Cancer Res 2015;75:1482-94.

3. Krause M, Dubrovska A, Linge A, Baumann M. Cancer stem cells: radioresistance, prediction of radiotherapy outcome and specific targets for combined treatments. Adv Drug Deliv Rev 2016 Feb 12. pii: S0169-409X(16)30052-7.

\section{A14}

\section{Cytokine profile involved in the maintenance of radioresistant prostate cancer stem cells}

\author{
Claudia Peitzsch ${ }^{1}$, Monica Cojoc ${ }^{1}$, Linda Hein ${ }^{1}$, Ina \\ Kurth $^{1,3}$, Anna Dubrovska1,2 \\ 'OncoRay-National Centerfor Radiation Research in Oncology, Dresden, Germany; \\ ${ }^{2}$ German Cancer Consortium (DKTK) Dresden, Dresden, Germany; \\ ${ }^{3}$ Nationales Centrum für Tumorerkrankungen (NCT) Dresden, Dresden, Germany
}

Aim: There is increasing evidence that human prostate cancer is driven by a malignant subpopulation with stem-like properties. These cancer stem cells (CSC) contribute to tumor-initiation, metastasis, therapyresistance and tumor relapse. We hypothesize that the determination of CSC-related biomarker in pretreatment biopsies of prostate cancer patients is correlating with treatment outcome and can be used for patient stratification and treatment selection. Methods: We generated isogenic radioresistant prostate cancer cell lines by applying several fractions of $4 \mathrm{~Gy}$ over a certain period of time until a total dose $>56$ Gy (RR). These radioresistant sublines exhibit higher expression of CSC marker (e.g. ALDH, CD133, CXCR4, ABCG2), epithelial-to-mesenchymal transition (EMT) phenotypes, higher self-renewal properties (sphere-formation), higher tumorigenicity and higher migratory activity. We applied several comparativeomic approaches, such as genomic, proteomic, metabolomic, epigenomic and secretome analysis, comparing aldehyde dehydrogenase (ALDH)-positive CSCs with the RR sublines to identify novel biomarker for prostate cancer radioresistance and to unravel the contributing molecular mechanisms. Results: Within our first proof-of-principle study, we could show that ALDH-positive CSCs are radioresistant and maintained directly by the $W n t / \beta$-catenin signaling pathway. ${ }^{[1]}$ In addition, we found that irradiation is inducing in a dose- and time-dependent manner several CSC marker and CSC properties. This irradiation-induced CSC-plasticity was attributed to the modulation of the histone methylation code. ${ }^{[2]}$ Within the presented study we analyzed a panel of secreted cytokines in the medium of the radioresistant sublines and found for example the CXCR4-CXCL12 signaling to be involved in the CSC maintenance and the induction of radioresistance in prostate cancer. This was proven in a s.c. xenotransplantation model in vivo and in ex vivo treated primary prostate cancer biopsies. Conclusion: The CXCR4-CXCL12 signaling axis is involved in the maintenance of prostate CSCs and is contributing to their radioresistant properties.

\section{Key words:}

Radiotherapy, cancer stem cells, cytokines, plasticity

\section{References}

1. Peitzsch C, Cojoc M, Hein L, Kurth I, Mäbert K, Trautmann F, Klink B, Schröck E, Wirth MP, Krause M, Stakhovsky EA, Telegeev GD, Novotny V, Toma M, Muders M, Baretton GB, Frame FM, Maitland NJ, Baumann M, Dubrovska A. An epigenetic reprogramming strategy to resensitize radioresistant prostate cancer cells. Cancer Res 2016;76:2637-51.

2. Cojoc M, Peitzsch C, Kurth I, Trautmann F, Kunz-Schughart LA, Telegeev GD, Stakhovsky EA, Walker JR, Simin K, Lyle S, Fuessel S, Erdmann K, Wirth MP, Krause M, Baumann M, Dubrovska A. Aldehyde dehydrogenase is regulated by $\beta$-Catenin/TCF and promotes radioresistance in prostate cancer progenitor cells. Cancer Res 2015;75:1482-94.

\section{A15}

\section{Oxidative stress, metastasis and melanoma stem cell - in vitro and in vivo analysis}

Telma Lisbôa-Nascimento, Milene Ormanji, Darcy Marinho, Michele Longoni Calió, Vera Lúcia Rigoni, Clélia Rejane Antônio-Bertoncini, Alice Teixeira Ferreira, Francisco Ribas Bosco

Federal University of Sao Paulo, Sao Paulo, Brazil

Aim: Cancer stem cells play an essential role to maintain the tumor size or fuel its growth. In an advanced stage of melanoma, the presence of a subpopulation of melanoma stem cells (MSC) is reflected in the resistance to the therapies and development of the metastasis. MSC exhibit an altered metabolism when compared to normal melanocyte. This alteration increases in the presence of reactive oxygen species (ROS), such as superoxide anion 
(O2._), which stimulating all three stages of cancer: initiation, promotion and progression. In this work, we analyzed the effects of superoxide anion in MSCs in vitro and in vivo. Methods: The study was approved by Ethical Committee of the Federal University of Sao Paulo, process number: 1523/2008. In this work, the melanoma lineage TM5, was cultured in long term under specific medium containing: bFGF, EGF, LIF and retinoic acid, giving rise to the MSC as we describe in literature. We analyze the viability, proliferation and survivability of MSCs through techniques such as MTT (3-(4, 5-dimethylthiazolyl-2)-2, 5-diphenyltetrazolium bromide), flow cytometry, neubauer camera using biomarkers as: $\mathrm{Brd}-\mathrm{U}, \mathrm{Bcl}-2$ and $\mathrm{Ki}-67$, for after, evaluate the presence of superoxide anion, through dihydroethidium (DHE) and its function in vitro and in vitro using the same mentioned techniques and confocal microscopy and immunohistochemistry. Results: Long-term culture induced the development of cells with some stem cells characteristics. We named these cells as melanoma stem cells; these form an adherent and nonadherent spheres as well as they showed an increased pigmentation. MSC possess an enhanced ability to survive and adapt after changing the culture medium (epigenetic effect). In vitro, MSC shows an outgrowth and quiescence state and self-renewal. MSC presented less expression of $\mathrm{Ki}-67$ when compared with melanoma control cells and both: MSC and control cells presents good viability. The levels of the O2._ in MSCs are increased significantly compared to the melanoma control cells, suggesting a possible protection against apoptosis in MSCs, because occurred a concomitant increase of expression of the anti-apoptotic protein Bcl-2 and these cells presented more survival capacity. In vivo, occurred a decrease in the levels of O2._, this reduction may be involved in increased of malignancy of melanoma. It was observed an increasing of the expression of the Ki-67 e decreased of the Bcl-2. The microenvironment, in vivo, could change the behavior of the MSC, increasing its proliferation and migration and invasion capacity to the neighboring tissues. Conclusion: These results indicate two different conclusions: In vitro, O2._ increases and protects MSC against apoptosis, and participates in the survival of these cells and differentiation process. In vivo, was observed the less expression of: O2._ and $\mathrm{Bcl}-2$, and the greater expression of $\mathrm{Ki}-67$, suggesting that in vivo, the O2._ play an important role in cellular proliferation due its interaction with the microenvironment, acting in ways that promotes progression and invasion tumor. Finally, our results imply that the different levels of O2._ acts in different signaling cascades to promote cell proliferation or differentiation.

\section{Key words:}

Superoxide anion, cancer stem cell, melanoma, oxidative stress

\section{A16 \\ Non-alcoholic fatty liver disease and risk for hepatocellular carcinoma - do cancer stem cells matter?}

\section{Jürgen Borlak}

Hannover Medical School, Hannover, Germany

Hepatocellular carcinoma (HCC) is a frequently diagnosed cancer worldwide and a leading cause of cancer mortality. This malignancy results primarily from viral liver disease, alcoholic injury, aflatoxins and to a lesser extent from genetic disorders such as hemochromatosis. Clinical epidemiology studies suggest an association between non-alcoholic fatty liver disease (NAFLD) and risk for liver cancer. Given the epidemic in fatty liver disease the risk for HCC appears to be particularly increased in NASH cirrhosis patients. ${ }^{[1]}$

The mechanisms leading to tumor growth in fatty liver disease are unknown, nonetheless may involve the complex interplay of adipokines and cytokines in promoting hepatocarcinogenesis. ${ }^{[2,3]}$ Importantly, research identified a decisive role of hepatic stem cells in the development of liver cancer. However, it remains enigmatic why stem cells become cancerous (CSC). Several landmark papers evidence dysregulation of signalling pathways in the control of self-renewal and differentiation of hepatic stem cells and include PI3K/ Akt, JAK/STAT, Wnt/ß-catenin, hedgehog, Notch, NF$\mathrm{KB}$ and $\mathrm{ABC}$ transporters to influence stemness of CSCs. ${ }^{[4,5]}$ Knowledge on these pathways permits the development of molecularly targeted therapies.

In my presentation I will report recent findings on the regulation of cancer stem cells in steatotic human hepatoma cells to mimic the condition of NAFLD. I will particularly focus on signalling pathways either linked to WNT and Hedgehog signalling, cell cycle regulation and chromatin organisation and provide an overview on the surplus of putative targets obtained from a wide range of cell biology, life cell imaging, genomics and computational biology studies. I will also discuss the possibilities for therapeutic intervention studies in preventing the induction of CSC in NAFLD patients and this includes some preliminary findings with dual kinase inhibitors obtained from the Botta-lab of Siena, Italy.

\section{Key words:}

Non-alcoholic fatty liver disease, hepatocellular 
carcinoma, steatotic hepatoma cells, tumor growth signalling pathways, dual kinase inhibitors

\section{References}

1. White DL, Kanwal F, El Serag HB. Association between nonalcoholic fatty liver disease and risk for hepatocellular cancer, based on systematic review. Clin Gastroenterol Hepatol 2012;10:1342-59.

2. Baffy G, Brunt EM, Caldwell SH. Hepatocellular carcinoma in non-alcoholic fatty liver disease: an emerging menace. $J$ Hepatol 2012;56:1384-91.

3. Sun B, Karin M. Obesity, inflammation, and liver cancer. $J$ Hepatol 2012;56:704-13

4. Chen K, Huang YH, Chen JL. Understanding and targeting cancer stem cells: therapeutic implications and challenges. Acta Pharmacologica Sinica 2013;34:732-40.

5. Yamashita T, Wang XW. Cancer stem cells in the development of liver cancer. J Clin Invest 2013;123:1911-8.

\section{A17}

Metastatic prostate cancer cells are endowed with cancer stem cell properties and interact with host cells to establish a proinflammatory microenvironment conducive to metastasis

Kiera Rycaj ${ }^{1}$, Hangwen $\mathrm{Li}^{2}$, Mahipal Suraneni ${ }^{2}$, Xin Chen ${ }^{1}$, Collene Jeter ${ }^{2}$, Tammy Calhoun-Davis ${ }^{2}$, Hseuh-Ping $\mathrm{Chao}^{2}$, Jianhua $\mathrm{Hu}^{2}$, Dean Tang ${ }^{1}$

${ }^{I}$ Roswell Park Cancer Institute, Buffalo, USA;

${ }^{2}$ MD Anderson Cancer Center, Houston, USA

Prostate cancer ( $\mathrm{PCa}$ ) is the second leading cancer in American men with an estimated 220,800 new cases and 27,540 deaths in 2015. Androgen deprivation therapy (ADT) is currently the mainstay for advanced $\mathrm{PCa}$ patients; unfortunately, most treated patients eventually develop the castrationresistant disease (CRPC). Like most other solid tumors, the worst outcome for PCa patients, whether treated or not, is development of distant metastasis. Although many PCa cell-intrinsic molecules and endorgan factors have been implicated in the metastatic dissemination of $\mathrm{PCa}$ cells, the role of primary tumor microenvironment and the nature of the metastatic $\mathrm{PCa}$ cells remain poorly defined. Here we attempt to address the two questions by combining experimental PCa metastasis models in NOD/SCID mice and CDNA microarray-based expression profiling followed by extensive functional studies. We first show that $\mathrm{PCa}$ cells implanted orthotopically (i.e. in the prostate) metastasize much more extensively and widely than those implanted ectopically (i.e. subcutaneously or s.c). Microarray-based gene expression profiling reveals that the orthotopically implanted human PCa cells upregulate several classes of genes that have been implicated in metastasis and include those involved in: proteolysis/invasion/angiogenesis, inflammation/ cytokine signaling, and developmental pathways/stem cell signaling. Remarkably, mouse-specific microarray analysis shows that several classes of host (mouse) genes, which include those related to myoepithelial and myofibroblast phenotype/cytoskeleton/motility, extracellular matrix/matrix remodeling, inflammation/ immune functions, and development/stem cells, are significantly upregulated in the orthotopic prostate tumors. These findings suggest that the implanted human PCa cells reciprocally interact with the host prostatic cells (both epithelial and non-epithelial) to establish a proinflammatory microenvironment highly conducive to $\mathrm{PCa}$ metastasis. Further, we provide multiple pieces of evidence that metastatic/ metastasizing $\mathrm{PCa}$ cells have cancer stem cell (CSC) properties. This data not only advances our understanding of the biology of PCa development and progression but also lays a foundation for developing novel therapeutics to target the tumor microenvironment as well as rare tumorigenic and metastasis-initiating CSCs.

\section{Key words:}

Prostate cancer, tumor microenvironment, metastasis, cancer stem cell

\section{A18}

\section{EGF/EGFR pathway is sufficient to induce aggressiveness and expression of pluripotency markers of patients-derived glioblastoma cells}

Fabien Almairac ${ }^{1,2}$, Laurent Turchi', Denys Fontaine ${ }^{2}$, Philippe Paquis ${ }^{1,2}$, Hervé Chneiweiss ${ }^{4}$, Marie-Pierre Junier ${ }^{4}$, Fanny Burel-Vandenbos ${ }^{1,3}$, Thierry Virolle ${ }^{1}$

${ }^{\prime}$ University Nice-Sophia Antipolis, CNRS, INSERM, institut Biologie Valrose, Nice, France;

${ }^{2}$ Neurosurgery Department, University Hospital of Nice, Nice, France; ${ }^{3}$ Pathology Department, University Hospital of Nice, Nice, France,

${ }^{4}$ University Pierre et Marie Curie, UMCR18, Neurosciences Paris Seine, Paris, France

Aim: Glioblastomas are the most devastating adult brain neoplasms. Recurrences are ineluctable, and presumed to be due to the glioma-initiating cells (GiCs). GiCs exhibit stem-cells properties such as selfrenewal and pluripotency, and are highly tumorigenic. EGFR is a hallmark of infiltrative gliomas, and is overexpressed in almost $50 \%$ of glioblastomas. Our objectives were to demonstrate that glioblastoma cells are able to interconvert from a differentiated state to a stem-like state and conversely depending on their environment, and to explore the underlying biological mechanisms, particularly the EGF/EGFR/ 
ERK pathway. Methods: Tumor cells were dissociated from freshly resected human glioblastomas. They were characterized for the stem-like markers (CD133, Sox2, Oct4, Nanog) as well as for the differentiation markers (GFAP) by immunostaining and flow cytometry methods. Functionally, cells were assessed for their clonal properties in vitro, temozolomide sensitivity in vitro, and tumorigenicity in vivo using orthotopical xenotransplantations on NOD/SCID mice. Results: Spontaneously, most of the cells harbored differentiated properties. Functionally, these cells did not have clonogenic properties, were sensitive to temozolomide (mean surviving cells $=20 \%$ ), and were not able to form a tumor in brain mice. After $48 \mathrm{~h}$ of culture in an EGF enriched medium, the differentiated cells acquired stem-like properties. They expressed Sox2/Oct4/Nanog, displayed long term self-renewal in a clonogenic single-cell assay, and became more resistant to temozolomide (mean surviving cells $=60 \%$ ). The xenotransplantation of few of these cells led to the development of large tumors after 3 months. Interestingly, the dedifferentiation process was quickly reversible, after only 4 days of culture in a serum medium. Also, we demonstrated that the de-differentiation process was inhibited by the adjunction of an anti-EGFR antibody (cetuximab) in the EGF medium, suggesting a pivotal role of the EGF/EGFR/ERK pathway. This pathway was strongly activated during the dedifferentiation process. Conclusion: The cellular plasticity concept contrasts with the unidirectional commitment originally described in the cancer stem cell model. It supplies a new comprehensive level on the CiGs' origins, on their resistance mechanisms to genotoxic stresses, and on the tumor heterogeneity. These results emphasize the importance of targeting specifically the stem-like properties of the tumor cells, not only the GiCs, to prevent further tumor enrichment in GiCs.

\section{Key words:}

Glioblastoma, cancer stem-cell, glioma initiating cells, plasticity, EGF, cetuximab

\section{A19}

\section{Cancer stem cell regulatory mechanisms change at late stages of skin squamous cell carcinoma progression}

Victoria da Silva-Diz ${ }^{1}$, Pilar Simón-Extremera ${ }^{1}$, Adrià Bernat-Peguera $^{1}$, Jana de Sostoa ${ }^{1}$, Maria Urpi ${ }^{1}$, Rosa M Penín ${ }^{2}$, Diana Pérez-Sidelnikova ${ }^{3}$, Oriol Bermejo ${ }^{3}$, Joan Maria Viñals ${ }^{3}$, Annie Rodolosse ${ }^{4}$, Eva GonzálezSuárez', Antonio Gómez-Moruno', Miguel Angel Pujana', Manel Esteller ${ }^{1}$, Alberto Villanueva', Francesc Viñals', Purificación Muñoz ${ }^{1}$
${ }^{I} I D I B E L L$, Barcelona, Spain;

${ }^{2}$ Pathology Service, Hospital Universitario de Bellvitge/IDIBELL, Barcelona, Spain;

${ }^{3}$ Plastic Surgery Unit, Hospital Universitario de Bellvitge/IDIBELL, Barcelona, Spain;

${ }^{4}$ Institute for Research in Biomedicine (IRB Barcelona), Barcelona, Spain

Cancer stem-like cells (CSC) play key roles in long-term tumor propagation and metastasis, but their dynamics during disease progression are not understood. Tumor relapse in patients with initially excised skin squamous cell carcinomas (SCC) is characterized by increased metastatic potential, and SCC progression is associated with an expansion of CSC. Here, we used genetically and chemicallyinduced mouse models of skin SCC to investigate the signaling pathways contributing to CSC function during disease progression. We found that CSC regulatory mechanisms change in advanced SCC, correlating with aggressive tumor growth, a strong induction of the EMT program and enhanced metastasis. $\beta$-catenin and EGFR signaling, induced in early SCC CSC, were downregulated in advanced SCC. Instead, autocrine FGFR1 and PDGFR $\alpha$ signaling, which have not been previously associated with skin SCC CSC, were upregulated in late CSC and promoted tumor growth and metastasis, respectively. Finally, highgrade and recurrent human skin SCC recapitulated the signaling changes observed in advanced mouse SCC. Collectively, our findings suggest a stage-specific switch in CSC regulation during disease progression that could be therapeutically exploited by targeting the PDGFR and FGFR1 pathways to block relapse and metastasis of advanced human skin SCC.

\section{Key words:}

Cancer stem cells, skin carcinoma, metastasis, EMT, PDGFR, FGFR

\section{A20}

\section{The role of $\alpha 2 \beta 1$ integrin on prostate cancer cell stemness}

Marjaana Ojalill' ${ }^{1}$ Elina Taipalus ${ }^{1}$, Johanna Jokinen ${ }^{1}$, Pekka Taimen ${ }^{1}$, Peter J. Boström ${ }^{2}$, Jyrki Heino ${ }^{1}$

${ }^{1}$ University of Turku, Turku, Finland;

${ }^{2}$ Turku University Hospital, Turku, Finland

Tumor microenvironment is acknowledged to be a critical component for tumor formation, consisting of cancer and stromal cells together with extracellular matrix (ECM). During tumor development the normal ECM is reorganized and new components synthesized by cancer associated fibroblasts (CAFs), this may create favorable conditions for cancer cell proliferation, invasion and a niche for cancer stem cells. In prostate 
epithelial/cancer stem cells are identified as CD44+, $\mathrm{CD} 133+, \alpha 2 \beta 1$ integrinhigh. Integrin $\alpha 2 \beta 1$ is a collagen receptor, which proposes that cell-collagen interaction is important for the stem cell biology in prostate. However the role of specific ECM proteins and cellular receptors in regulating prostate stem cells remains poorly understood.

Here, we have used primary cultures of prostatederived fibroblastic cells, allowed them to generate ECM, these matrices and specific matrix proteins (collagen I and fibronectin) were used to examine prostate cancer cell-line DU145 proliferation and drug resistance to widely used anti-mitotic chemotherapy drug docetaxel. Sorting cells to sub-populations based on their expression of $\alpha 2 \beta 1$ integrin allowed us to investigate whether higher expression of $\alpha 2 \beta 1$ integrin is in consistence with theory that cells with high $\alpha 2 \beta 1$ integrin are more resistant to cytotoxic drugs. To study thoroughly the role of $\alpha 2 \beta 1$ integrin on prostate cancer stem cells, we created $\alpha 2$ integrin knock-out of DU145 cell-line by CRISPR/Cas 9 system and compared it with wild-type DU145 cells and rescued cells, which were transfected with plasmid carrying $\alpha 2$ integrin construct.

Our results present that ECM-cancer cell interaction reduced proliferation of cancer cells however according to EC50 values had no effect on the resistance to docetaxel. The EC50 values on collagen I were 18.8 \pm 0.3 ; on fibronectin: $19.5 \pm 0.2$; on fibroblast-derived ECM: $19.6 \pm 0.3$. Based on collected data we concluded that there was no protective feature of fibroblastderived ECM to DU145 cancer cells from docetaxel induced cell death. Cells that survived docetaxel treatment presented significantly higher expression of a2 integrin and CD44, suggesting enrichment of stemlike cell population. Indeed, the sub-population with high expression of $\alpha 2 \beta 1$ integrin had slightly better survival rates. The ongoing study on whole genome sequencing of CRISPR/Cas9 modified DU145 cells will reveal the differences in gene expression and may bring to light some new properties of integrin signaling.

\section{Key words:}

Prostate cancer stem cells, $\alpha 2 \beta 1$ integrin, drug resistance, CRISPR/Cas9 system, extracellular matrix, cancer associated fibroblasts

\section{A21 \\ YAP/TAZ, transcription factors at the roots of cancer}

Luca Azzolin, Michelangelo Cordenonsi, Stefano Piccolo

Department of Molecular Medicine, University of Padova, Padova, Italy
Tumors are complex tissues and cancer is a disease characterized by aberrant differentiation as much as it is of disturbed proliferation. Tumor cells are phenotypically plastic, and an unsolved issue in cancer biology is to what extent the expansion of cancer stem cells representation that accompany tumor progression is caused by expansion of preexisting stem cells or, rather, by a differentiation block or even de-differentiation of more differentiated tumor cells. Indeed, the molecular mechanisms that preserve differentiation or induce cell plasticity in neoplastic or normal tissues, as in the case of acquisition of stem-cell traits by more mature cells during tissue repair, remain unknown. At this meeting, I will present new evidence indicating the role of YAP eTAZ, transcriptional effectors of Hippo- mechano- and Wnt-signaling, in regulating cell plasticity. It appears that these properties of YAP/ TAZ are independent of acquisition of a mesenchymal phenotype, require interaction with chromatin and are shared by multiple cell type, including non-epithelial ones. Notably, in mouse models, YAP/TAZ are essential for normal stem cells of breast, pancreas and neural tissues when these are activated by tissue damage in vivo, or for growth as organoids ex vivo. In these are other tissues, this correlate with the genetic requirement of YAP/TAZ to initiate tumorigenesis. The modalities of YAP/TAZ regulation by Wnt and other upstream cues will be also discussed.

\section{Key words:}

YAP/TAZ, cancer stem cells, Wnt signalling, cell plasticity

\section{A22 \\ Use of 3D spheroid cultures to screen for drugs targeting cancer stem cells}

\author{
Juan Gumuzio, Olatz Leis, Angel G. Martin
}

StemTek Therapeutics, Derio, Spain

The cancer stem cell (CSC) concept has important implications not only for our understanding of carcinogenesis, but also for the development of cancer therapeutics. There is a growing body of preclinical evidence showing that cancer stem cells contribute to chemotherapy and radiation resistance in breast cancer. The use of drugs that interfere with stem cell self-renewal represents the strategy of choice for novel effective anti-cancer treatments, but also a great challenge because cancer stem cells and their normal counterparts share many pathways.

The biology of cancer stem cells has proven complex and difficult to translate into effective therapeutic 
strategies. In order to monitor the effect of test compounds on cancer stem cells, a panel of markers, preferably easy-to-measure surface markers, must be defined. This is, however, cumbersome, since for many tumor indications that marker panel is not clearly defined, with often non-overlapping combinations of markers defining cell populations with cancer stem cell activities or tumor initiation ability. This is most likely reflecting the changing nature of the stemness capacity in tumor cells.

Thus, the question arises as: how do we test compounds for anti-cancer stem cell activity? The answer is: phenotypic screening. There are indeed several functional assays well validated in the scientific literature that have been used for years associated to the ability of cancer cells to demonstrate stem cell behavior. The most relevant is the $3 \mathrm{D}$ tumor spheroid assay. This assay has been used to uncover and culture stem cells from many tissues as well as from tumors. There are multiple reports now that show that spheroid derived cells are enriched in tumor initiating or cancer stem cells, derived from cell lines and from natural fresh tumors as well. There are several conceptual considerations that need to be taken into account in order to apply this assay to cancer stem cells:

1. This assay may be used to assess stem cell content only when 3D spheroids are formed through cell growth from single cells, not by aggregating cells. Aggregation does not impose the growth restriction necessary to allow stem cells to differentially survive.

2. Not every tumor and cell line is amenable to spheroid growth. This is related to cell adhesion expression profile, as some cells will not form spheroids but disperse cell clusters. Therefore only validated cell lines may be used on the spheroid assay.

3. Care must be taken with cytotoxic compounds. A non-specific cell toxic drug will kill cells regardless of culture conditions, thus only non-toxic concentrations may be used.

The tumor spheroid assay can measure stem cell number in the parental population, by looking at the number of spheres obtained or sphere forming cell frequency through limiting dilution assay. But it can also measure effect on stem cell proliferation by measuring sphere diameter upon time.

Here we describe the use of 3D spheroid models to profile compound activity against cancer stem cells. Furthermore, a case of compounds preventing hypoxia-inducible transcription factor (HIFs) activity is presented. Recently, HIF transcription factor biology has been linked to pathways that regulate stem cell selfrenewal and pluripotency, suggesting a new mechanism whereby HIF proteins may drive tumor growth, through the generation of tumour-initiating cells or cancer stem cells. Therefore, targeting the HIF pathway may provide a novel therapeutic avenue to target cancer stem cells. We demonstrate that interfering with HIF pathway activation prevents mammosphere formation, validated through indenpendent confirmation through Sox2 promoter activation, Aldefluor ${ }^{\circledR}$ assay and in vivo proof-of concept experiments targeting tumor initiation.

The assays performed in this work are part of StemTek Therapeutics portfolio of research services, specialized in targeting cancer stem cells for drug discovery.

\section{Key words:}

Cancer stem cells, 3D spheroids, drug development

\section{A23 \\ CD34+ cells in blood and primary tumor foci in head and neck squamous cell carcinoma patients receiving radiotherapy}

\section{Elena Selivanova, Vyacheslav Andreev, Sergey Makarenko, Irina Zamulaeva}

A. Tsyb Medical Radiological Research Centre - branch of the National Medical Research Radiological Centre of the Ministry of Health of the Russian Federation, Obninsk, Russia

The aim of this work was to elucidate the regularities of changes in the frequency of CD34+ hematopoietic stem cells (HSCs) circulating in peripheral blood and accumulating in primary tumor focus in patients with head and neck squamous cell carcinoma (HNSCC) after their exposure to the low-LET ionizing radiation receiving radiotherapy.

A study group consisted of 35 patients with HNSCC in age from 44 to 85 years (mean age $59.5 \pm 1.3$ years). The frequency of HSCs were determined in patients' biopsies and blood cell samples before treatment and at $24 \mathrm{~h}$ after receiving of the subsequent local dose of $\mathrm{Y}$-irradiation of $10 \mathrm{~Gy}$ with conventionally fractionated dose of 2 Gy daily. Stem cell viability and a number of HCS were evaluated in the disrupted and dissociated tumor pieces and in blood cell samples by three-color FACS analysis using DNA binding dye Hoechst 33342 and anti-CD45 and CD34 antibodies.

The high individual variability in the frequency of CD45lowCD34+HSCs was found in the tumor biopsies and blood obtained from patients before treatment. The median frequency of CD34+ cells before treatment was in the blood of 4.9.10-4, in biopsy material is 1.6.10-2. 
This indicates a significant accumulation of the studied cells in the primary tumor. Correlation between initial frequency of the HSCs and clinical-morphological characteristics of the tumor was not observed There was a tendency to the decreased frequency of CD34+ cells in the blood samples to 3.6.10-4 $(P=0.1$ in comparison with the pretreatment frequency) after irradiation. High statistically significant correlation was observed at the individual level between the frequency of these cells before and after exposure $(R=0,82$, $P<0.0001)$. The median frequency of CD34+ cells in the tumor, in contrast, was increased to 2.7.10-2 and did not correlate with the initial frequency of these cells before treatment in this group of patients. The results obtained indicate the decrease in migration of hematopoietic cells after the first sessions of radiation therapy, probably, due to the reduction of signals produced by the tumor to the chemotaxis and mobilization of these cells from the bone marrow. The regulation of the number of intra-tumoral hematopoietic cells is a complex process, including their differentiation in different types of cells. It is known that ionizing radiation disturbs the differentiation process of hematopoietic cells which may be to a cause of the observed increase in the frequency CD34 + cells in the majority of HNSCC patients. In the future, it would be of great interest to evaluate the predictive value of the number of CD34 + cells before treatment and changes in this index during radiotherapy.

\section{Key words:}

Head and neck squamous cell carcinoma, low-LET ionizing radiation

\section{A24}

\section{The therapeutic targets of miRNA in hepatic cancer stem cells}

\section{Sabrina Bimonte, Maddalena Leongito, Antonio Barbieri, Francesco Izzo}

IRCCS Istituto Nazionale dei Tumori Fondazione G. Pascale, Napoli, Italy

Hepatocellular carcinoma (HCC) is the fifth most common cancer worldwide malignancy, and the third leading causes of cancer death in patients. Several studies demonstrated that hepatic cancer stem cells (HCSCs), also called tumor-initiating cells, are involved in the regulation of $\mathrm{HCC}$ initiation, tumor progression, metastasis development and drug resistance. Despite the extensive research, the underlying mechanisms by which HCSCs are regulated remain still unclear. MicroRNAs (miRNAs) are able to regulate several biological processes such as self-renewal and pluripotency of HCSCs, representing a new promising strategy for treatment of HCC chemotherapy-resistant tumors. In this review, we synthesize the latest findings on therapeutic regulation of HCSCs by miRNAs, in order to highlight the perspective of novel miRNA-based anticancer therapies for HCC treatment.

\section{Key words:}

Hepatic cancer stem cells, hepatocellular carcinoma, signaling pathway, microRNA

\section{A25}

\section{Targeting metalloproteinases to sensitize breast cancer stem cell to radiation}

María Auxiliadora Olivares-Urbano', Sandra RíosArrabal $^{1,2}$, María Escarlata López ${ }^{3}$, María Isabel Núñez ${ }^{1,2,4}$

${ }^{I}$ Department of Radiology and Physical Medicine, University of Granada, Granada, Spain;

${ }^{2}$ Institute of Granada (ibs.GRANADA), University Hospitals of GranadaUnivesity of Granada, Granada, Spain,

${ }^{3}$ Department of Radiation Oncology, Oncosur, Granada, Spain,

${ }^{4}$ Biopathology and Medicine Regenerative Institute (IBIMER), University of Granada, Granada, Spain

Aim: The use of adjuvant or neoadjuvant radiotherapy improves local control and survival rate while maintaining normal tissue toxicities at acceptable levels. Nonetheless, cell radiation resistance is a limiting factor for this treatment. Radioresistance is due, in part, to cancer stem cells (CSCs) not killed by radiation. Thus CSCs isolation from breast tumours will be important for a better understanding of molecular mechanisms involved in their origin, self-renewal, differentiation into tumour cells, resistance to radioand chemotherapy, invasiveness and metastatic ability. Methods: Two breast cancer cell lines (MCF7 and MDA-MB-231) were maintained in monolayer culture and then cultured in sphere medium. At $24 \mathrm{~h}$ after seeding in monolayer, cells were irradiated at 2, 4 or $6 \mathrm{~Gy}$, maintaining a non-irradiated control. From the general population of cells cultured in sphere media, positive and negative CSCs were separated with flow cytometry. Stem cell markers considered were ALDH1, CD24 and CD44. The genes measured in both positive and negative sub-populations were: MMP-1, MMP-2, MMP-3, MMP-9, MMP-13, HDAC-1, HDAC-2, HDAC-4, TIMP-1 and TIMP-2. Results: After ionizing radiation treatment, the proportion of CSCs markers varied according to the dose and cell type. ALDH positivity was increased at $4 \mathrm{~Gy}$ in MCF-7 cells, while ALDH1 and CD44 were increased at 6 Gy in the MDAMB-231 line. More genes were expressed in the MDAMB-231 versus MCF-7 line. MMP-1, MMP-2, HDAC-4, and TIMP-1 genes were expressed in both cell lines. MMP-3, MMP-9, MMP-13, HDAC-2 and TIMP-2 were 
also expressed In MDA-MB-231 cells. In general, genes were more expressed in the negative population than in the positive, highlighting the positive CSCs expression of MMP-2 and MMP-9, which are related to the formation of secondary tumors. Conclusion: Radiation enhances the population of CSCs in different ways according to the dose and cell line. Post-radiation, a larger number and higher proportion of genes were expressed in the MDA-MB-231 cell line, which is more radioresistant in comparison to MCF-7. The increased MMP-2 and MMP-9 expression after radiation would contribute to modifying the cell survival capacity and differentiation status of MDA-MB-231 cells and may correspond to a phenotype linked to carcinogenesis. The increase in this expression with higher radiation dose in the positive CSC population may play a role in the development of secondary tumors, facilitating ECM degradation by the cells that survive radiation. Administration of inhibitors of these MMPs to patients undergoing radiotherapy may be useful to avoid the radiation-induced development of a more aggressive phenotype that promotes tumor progression. Both MMP-2 and MMP-9 may be considered novel therapeutic targets in cancer treatment.

Key words:

CSCs, ionizing radiation, MMPs, breast cancer, HDAC

\section{A26}

CD57 defines a novel maker of glioblastoma stem cells that have greater invasive potential than CD133+ tumor cells

Lin Qi, Yulun Huang, Mari Kogiso, Hua Mao, Holly Lindsay, Patricia Baxter, Jack Su, Laszlo Perlaky, Ching Lau, Murali Chintagumpala, Xiao-Nan Li

Baylor College of Medicine, Houston, USA

Glioblastoma multiforme (GBM) is the most aggressive and lethal brain tumor that occurs both in children and adults. Diffuse invasion into normal brain tissue is one of the important biologic features that make GBM refractory to conventional therapies. While existing studies on GBM invasion are primarily conducted using tumor core tissues from surgical resections, it is unclear whether unresectable, infiltrative GBM cells would be more informative for studying their invasive nature compared to those in the resected tumor cores. More importantly, little is known if and which cancer stem cell populations are driving glioma invasion. To address these fundamental issues, we utilized our panel of 7 (6 pediatric and 1 adult) patient tumorderived orthotopic xenograft mouse models of GBM to isolate invasive GBM cells (infiltrating normal mouse brain parenchyma) and tumor core GBM cells and directly compared their biological differences. Our result showed that the invasive cells have stronger neurosphere forming efficiency in vitro in a serial dilution assay and increased tumorigenic capacity after in vivo transplantation (particularly at 100 cells/ mouse) compared to the tumor core cells. A screening of putative cancer stem cell markers (CD133, CD15, CD24/CD44, CD57 and CD117) showed that invasive GBM cells are enriched ( $>2$ folds) with CD57+ cells compared to the tumor core cells, and these infiltrating cells were predominantly CD57+/CD133-. Even the CD133+ cells were frequently dual-positive with CD57 $(\mathrm{CD} 33+\mathrm{CD} 57+)$, not only in the xenograft tumors but also in a separate set of patient GBM samples. Mechanistically, we found that CD57+ cells expressed high levels of self-renewal genes and tend to stay in G0/G1 phases. In conclusion, we showed that invasive GBM cells are biologically deferent from the matched tumor core cells and identified CD57 as a novel stem cell marker that is associated with GBM infiltration. Our findings suggest that new anti-invasion therapies should target CD57+ cells in addition to CD133+ cells in GBM.

Key words:

CD57, GBM, xenograft

\section{A27}

Investigating radiation induced changes
in stem cell population of non-small cell
lung cancer (NSCLC) models following
stereotactic ablative radiotherapy (SABR)

\author{
Charlene Junkin ${ }^{1}$, Victoria Dunne ${ }^{1}$, Gerry Hanna ${ }^{1,2}$, Kevin \\ Prise $^{1}$, Karl Butterworth ${ }^{1}$
}

${ }^{I}$ Queen's University Belfast, Belfast, UK;

${ }^{2}$ Clinicial Oncology, Northern Ireland Cancer Centre, Belfast, UK

SABR is emerging as a powerful clinical technique for the treatment of localised and inoperable NSCLC. Despite improvements using radical chemoradiotherapy, overall 5 year survival rates remain low at between $7-20 \%$, better strategies are urgently needed to improve control rates. Radiotherapy failure can be attributed to resistance as a result of heterogeneity within the tumour which may include a sub-population of cancer stem cells (CSCs), these may impact the ability of the tumours to recur following radiotherapy. Furthermore, radiation exposure may impact CSC populations causing differentiated cells to acquire stem-like properties or normal stem cells to transform into CSCs due to genetic alteration or changes in the normal stem cell microenvironment. The underlying 
process of radiation induced stemness in NSCLC remains to be fully understood and may present novel opportunities for therapeutic intervention in combination with SABR that could potentially improve overall survival in NSCLC.

In this study, a panel of NSCLC cell lines (A549, H460, $\mathrm{H} 157$ ) were exposed to total doses of 6,12 and $24 \mathrm{~Gy}$ delivered in 3 fractions over a 9 day period using a XRAD 225 X-ray generator (PXI, Inc.). Flow cytometry analysis of the irradiated cell populations showed significant dose dependent increases in the populations of cells bearing the putative stem cell markers CD44 and CD133. This effect was greatest in the A549 cell model which showed a population increase of 9.8 , 13.9 and $24.7 \%$ at corresponding doses of 6,12 and $24 \mathrm{~Gy}$. We have identified interferon regulatory factor-7 (IRF-7) as a potential mediator of radiation induced plasticity and resistance. Quantitative PCR revealed a tumour-specific change in IRF-7 RNA expression upon irradiation, with a general relative increase in expression with increasing radiation dose. These data were compared with a normal bronchial epithelial cell model, which contrastingly showed a relative decrease in IRF7 RNA expression levels upon increasing doses of radiation. Flow cytometry analysis and IRF7 RNA quantification was repeated using the A549 model with the addition of acute hypoxia at the time of irradiation. I have not found a significant difference in the percentage of cells expressing these stem cell markers upon addition of hypoxic conditions, however, IRF-7 RNA expression levels appear to decrease by nearly $40 \%$ upon addition of hypoxic conditions without irradiation.

Our results demonstrate radiation induced cellular plasticity following exposure to hypofractionated schedules in models of NSCLC with a potential role of IRF-7. Ongoing work in our laboratory seeks to further investigate the underlying radiobiological mechanisms of CSC plasticity and the role of both acute and chronic hypoxia. We ultimately aim to identify novel therapeutic strategies, to prevent acquisition of stem-like properties, reduce treatment failure and improve outcomes.

\section{Key words:}

Cancer stem cells, stereotactic ablative radiotherapy, non-small cell lung cancer, CD133, CD44, interferon regulatory factor 7

\section{A28}

The JNK-STAT3 pathway as a therapeutic target in neuroblastoma

Mayumi Higashi
Kyoto Prefectural University of Medicine, Kyoto, Japan

Aim: Neuroblastoma is one of the most challenging tumours in children, and the 5-year survival rate of progressive neuroblastoma remains around $30-40 \%$ regardless of improvement of intensive therapies. We studied signal transduction cascades as targets for neuroblastoma therapy. It has been reported that one of major MAPKs JNK regulates STAT3, which is another pathway of STAT3 activation different from JAK-STAT3, by phosphorylating a specific amino acid residue. It has been reported that JNK or STAT3 maintains the stemness of cancer cells, although the role of this pathway is not clearly revealed. In our study, we found remarkable anti-tumour effects of inhibiting JNK-STAT3 pathway, by disrupting the stemness of neuroblastoma. Methods: Neuroblastoma cell lines IMR5, NLF and SK-N-AS were treated with JNK inhibitor SP600125 or JNK-IN-8, or STAT3 inhibitor Niclosamide in various concentrations. Cell viability was analysed using CellTiter (Promega). Immunofluorescence was performed with $\beta$-III tubulin antibody (SIGMA) to stain neural processes. Gene expression was analysed by THUNDERBIRD SYBR-Green real-time PCR system (TOYOBO). Results: Phosphorylation of STAT3 serine 727 , but not tyrosine 705 , was suppressed by JNK inhibitor SP600125 or JNK-IN-8, dose-dependently. JNK inhibitors SP600125, JNK-IN-8 and a STAT3 inhibitor Niclosamide induced resembled effects on neuroblastoma cells, although the effects were cell growth dependent. All inhibitors induced remarkable decrease in cell viability in fast proliferating cell lines IMR5 and NLF, while they induced deceleration of proliferation and cell differentiation with increased $\beta$-III tubulin staining of neural processes in slower proliferative SK-N-AS. Low dose of Niclosamide also induced neural differentiation in any cells. Expressions of cancer stem cell markers SOX2 and CXCR4 were decreased by treatment with inhibitors. Conclusion: Inhibition of JNK-STAT3 led cells to differentiation or cell death, which indicates the disruption of cancer stemness in neuroblastoma cells. Our findings show that inhibition of JNK or STAT3 is an ideal way of tumour suppression induced by disrupting homeostasis of cancer cells. Our results indicate that JNK-STAT3 pathway is a promising target for the novel treatment of neuroblastoma.

\section{Key words:}

JNK STAT3 neuroblastoma

\section{A29}

The role of secreted frizzled-related protein 4 (sFRP4) in chemo-sensitisation of cancer stem cells 
Abhijeet Deshmukh1, Frank Arfuso', Philip Newsholme², Arun. M. Dharmarajan' ${ }^{1}$

${ }^{1}$ Stem Cell and Cancer Biology Laboratory, School of Biomedical Sciences, Curtin Health Innovation Research Institute, Curtin University, Perth, Australia; ${ }^{2}$ School of Biomedical Sciences, Curtin Health Innovation Research Institute, Curtin University, Perth, Australia

Background: Cancer stem cells (CSCs) are the unipotent cell population present within the tumour mass. CSCs are known to be highly chemo-resistant and, in recent years, have gained intense interest as key tumour-initiating cells that play an integral role in cancer recurrence following chemotherapy. Aim: The study investigates molecular signals essential to sustain CSCs and target their activity using secreted frizzled-related protein 4 (sFRP4) alone or in combination with chemotherapeutic drugs. Methods: Cancer stem cells isolation: CSCs isolated from Breast (MDA231/MCF7), Ovary (A2780 P/ADR/Cis), and Prostrate (PC3/LnCap) tumour cell lines in serumfree conditions and enriched with growth factors (EGF/ FGF/B-27). Chemo-sensitisation/drug treatment: Sensitisation with sFRP4 was performed by adding sFRP4 to the CSCs culture alone or in combination with chemotherapeutic agents (Doxorubicin/Cisplatin) for $24 \mathrm{~h}$. Viability assay: MTT based was used according to the manufacturer's protocol to measure cell metabolic viability. Cell surface markers: To assist in determining their identity, cell surface markers (CD44 ${ }^{+}$I CD24 $/ \mathrm{CD} 133^{+}$) were examined in both monolayers and CSCs by flow cytometry, using CellQuest data acquisition and analysis software. RNA isolation and cDNA synthesis: Total RNA was isolated from cells using TRIzol reagent followed by chloroform extraction, isopropanol precipitation, and a $75 \%$ (v/v) ethanol wash and further transcribed into cDNA using a High Capacity cDNA kit. Western blotting: Total proteins were extracted from cells using RIPA denaturing buffer. The protein extracts were estimated using BCA Kit and $20 \mu \mathrm{g}$ of proteins were separated by $12 \%$ SDSPAGE and transferred onto a nitrocellulose membrane. Immunoblotting was performed by blocking the membrane in 5\% Non-Fat Dry Milk (NFDM) solution and incubating the membrane in 5\% NFDM/BSA containing primary antibodies overnight at $4{ }^{\circ} \mathrm{C}$. The membranes were incubated in 3\% NFDM containing secondary antibodies for $1 \mathrm{~h}$ at RT after three washes with PBS containing $0.1 \%$ Tween 20 . Signals were detected on a Chemi-Doc imaging analyser using ECL Western Blotting Substrate. Caspase assay: The intracellular levels and activation of caspase-8 and caspase-3 were followed by Western blotting using antibodies specific for the proenzymes and activated species. Caspase-3 activity was measured using the EnzChek Caspase-3 Assay Kit II (Molecular Probes,
Invitrogen). Results: The MTT assays conducted showed the chemo-sensitisation effect of sFRP4 when used in combination with tumour-specific drugs. The post-transcription data (Gene-Expression) collected from CSCs that have undergone combinatorial treatment with sFRP4 and chemotherapeutic drugs suggests there is downregulation of drug transporters and upregulation of angiogenic/apoptotic/cell death markers. The post-translational modification (protein expression) of CSCs shows the chemo-sensitisation effect of sFRP4, when used in combination with tumourspecific drugs, by downregulating the cell-survival and oncogenes signals and upregulation pro-apoptotic signals. In tumour cell lines, sFRP4 in combination with doxorubicin/Cisplatin, reduced the proliferative capacity of CSC population in vitro. Conclusion: Wnt/ $\beta$-catenin signalling is important for proliferation and self-renewal of CSCs in association with human tumorigenesis. The silencing of this signalling pathway by the application of sFRP4 suggests potential for improved in vivo chemo-responses.

\section{Key words:}

Cancer stem cells, chemo-sensitisation, Wnt signalling pathway, Wnt antagonist, secreted frizzled related protein 4

\section{A30}

\section{Induction of radioresistance and cisplatin resistance in HNSCC cell line after ionizing radiation}

\begin{abstract}
Vesna Todorovic ${ }^{1}$, Ajda Prevc ${ }^{1}$, Martina Niksic Zakelj ${ }^{1}$, Blaz Groselj ${ }^{1}$, Primoz Strojan', Maja Cemazar ${ }^{1,2}$, Gregor Sersa ${ }^{1}$
\end{abstract}

${ }^{I}$ Institute of Oncology Ljubljana, Ljubljana, Slovenia;

${ }^{2}$ University of Primorska, Faculty of Health Sciences, Izola, Slovenia

Head and neck squamous cell carcinoma (HNSCC) constitutes approximately $6 \%$ of all cancers worldwide. The risk of HNSCC is strongly associated to habitual exposure to tobacco or alcohol. In addition, oropharyngeal SCC (OPSCC) can arise also from infection with human papilloma virus (HPV). Management of HNSCC is complex and is in part correlated to risk factors. Namely, HPV-positive OPSCC has a greater response to radiation or chemoradiation than tobacco/alcohol related HNSCC. In addition, a significant problem of HNSCC is its recurrence, specifically in previously irradiated areas, due to induced radioresistance and radiation tolerance limits of already irradiated normal tissues.

The aim of our study was to investigate the response of three HNSCC cell lines to ionizing radiation and 
exposure to cisplatin in vitro. DNA damage was evaluated in all cell lines in response to ionizing radiation by quantification of $\mathrm{H} 2 \mathrm{AX}$ foci. HNSCC cell lines used in the experiments were $\mathrm{FaDu}$, a HPVpositive cell line 2A3, derived from FaDu cells, and a radioresistant cell line FaDu-RR, established from FaDu cells by repeated exposure to ionizing radiation. Radioresistant FaDu-RR cells were recovered after exposure to fractionated ionizing radiation (a total dose of $120 \mathrm{~Gy})$.

The selected HNSCC cell lines responded differently to ionizing radiation and cisplatin. FaDu-RR cells were the most radioresistant with a dose modifying factor (DMF) at $\mathrm{ED}_{50} 1.66$ compared to FaDu cells. Contrary, a HPV-positive $2 \mathrm{~A} 3$ cells were more sensitive to ionizing radiation than FaDu cells (DMF at $E_{50}$ 0.47). In addition, FaDu-RR cells also displayed crossresistance to cisplatin (1.8-fold potentiation in $\mathrm{IC}_{50}$ value) compared to FaDu cells, whereas $2 \mathrm{~A} 3$ cells were more sensitive to cisplatin compared to $\mathrm{FaDu}$ cells (2.9-fold reduction in $\mathrm{IC}_{50}$ value).

DNA damage after ionizing radiation was quantified by detection of $\mathrm{H} 2 \mathrm{AX}$ foci, which correlates to DNA double strand breaks. In all three cell lines, a peak of $\mathrm{yH} 2 \mathrm{AX}$ foci was detected as early as $30 \mathrm{~min}$ after ionizing radiation. Interestingly, at this time point a maximum number of $\mathrm{YH} 2 \mathrm{AX}$ foci was detected in radiosensitive $2 A 3$ cells (more than 20/nuclei), whereas in FaDu cells $11 \mathrm{yH} 2 \mathrm{AX}$ foci/nuclei were detected. In radioresistant FaDu-RR cells, only $7 \mathrm{yH} 2 \mathrm{AX}$ foci/nuclei were detected $30 \mathrm{~min}$ after ionizing radiation. The level of $\mathrm{YH} 2 \mathrm{AX}$ foci $24 \mathrm{~h}$ after ionizing radiation was reduced in all cell lines, however only in radioresistant FaDu-RR cells the number of residual $\mathrm{YH} 2 \mathrm{AX}$ foci was similar to the level of $\mathrm{yH} 2 \mathrm{AX}$ foci in unirradiated cells. In FaDu and $2 \mathrm{~A} 3$ cells, the number of residual $\mathrm{yH} 2 \mathrm{AX}$ foci was reduced approximately by half.

A different response to ionizing radiation and exposure to cisplatin was observed in tested HNSCC cell lines. Namely, repeated exposure to ionizing radiation induced radioresistance and resistance to cisplatin in FaDu-RR cell line, whereas 2A3, a HPV-positive cell line, were more sensitive to ionizing radiation and cisplatin compared to parental FaDu cell line. The observed differences in radiosensitivity of HNSCC cell lines can be at least partly contributed to induction and clearance of $\mathrm{yH} 2 \mathrm{AX}$ foci after ionizing radiation indicating differences in DNA damage repair.

\section{Key words:}

Radioresistance, head and neck, squamous cell carcinoma, chemoresistance, cisplatin, H2AX foci 